\title{
Near-threshold photoelectron holography beyond the strong-field approximation
}

\author{
XuanYang Lai, ${ }^{1}$ ShaoGang Yu, ${ }^{1,2}$ YiYi Huang, ${ }^{1,2}$ LinQiang Hua, ${ }^{1}$ Cheng Gong, ${ }^{1}$ Wei Quan, ${ }^{1}$ C. Figueira de Morisson Faria, ${ }^{3, *}$ \\ and XiaoJun Liu ${ }^{1, \dagger}$ \\ ${ }^{1}$ State Key Laboratory of Magnetic Resonance and Atomic and Molecular Physics, Wuhan Institute of Physics and Mathematics, \\ Chinese Academy of Sciences, Wuhan 430071, China \\ ${ }^{2}$ School of Physics, University of Chinese Academy of Sciences, Beijing 100080, China \\ ${ }^{3}$ Department of Physics and Astronomy, University College London, Gower Street, London WC1E 6BT, United Kingdom
}

(Received 12 August 2016; published 17 July 2017)

\begin{abstract}
We study photoelectron angular distributions (PADs) near the ionization threshold with a newly developed Coulomb quantum-orbit strong-field approximation (CQSFA) theory. The CQSFA simulations exhibit an excellent agreement with the result from the time-dependent Schrödinger equation. We show that the low-energy fan-shaped pattern in the PADs corresponds to a subcycle time-resolved holographic structure and stems from the significant influence of the Coulomb potential on the phase of the forward-scattered electron trajectories, which affects different momenta and scattering angles unequally. Our work provides a direct explanation of how the fan-shaped structure is formed, based on the quantum interference of direct and forward-scattered orbits. Moreover, our work shows that the fan-shaped pattern can be used to extract information on the target structure, as the number of fringes in the PADs depends strongly on the symmetry of the electronic bound state.
\end{abstract}

DOI: 10.1103/PhysRevA.96.013414

\section{INTRODUCTION}

Quantum interference of matter waves lies at the heart of quantum mechanics. When an atom or a molecule interacts with a strong laser field, the bound electron may be ionized by tunneling through the barrier formed by the Coulomb potential and the laser electric field [1]. The electron wave packets ionized at different times with the same final momentum will interfere with each other [2]. This results in rich interference patterns in the above-threshold ionization (ATI) photoelectron angular distributions (PADs) [3], which have been taken as an important tool in exploring the structure and the dynamics of atoms and molecules with attosecond temporal resolution and angstrom spatial resolution [4,5].

Recently, a new type of wave-packet interference, i.e., photoelectron holography [6-9], has provided a novel avenue for ultrafast retrieval of structural and dynamical information about the atomic or molecular medium. By analogy with optical holography [10], the electron wave packet which directly drifts to the detector after tunneling ionization is taken as a reference wave, while the electron wave packet which further interacts with the core and then drifts to the detector acts as a signal wave. These two paths with the same final momentum interfere with each other, forming the holographic patterns in the PADs. Since the signal wave scatters off the target and encodes its structure, the hologram stores spatial and temporal information about the core and electron dynamics. For example, a spiderlike holographic structure was experimentally observed in the PADs of metastable xenon atoms $[6,7]$. This specific structure is produced by the direct electron wave packets and the laser-driven forward-scattered electron wave packets from the same quarter cycle of the laser pulse; thus, subcycle time resolution is encoded in the holographic patterns [6,7]. Furthermore, signal and reference waves can

\footnotetext{
*c.faria@ucl.ac.uk

${ }^{\dagger}$ xjliu@wipm.ac.cn
}

be born in different quarter cycles, leading to different holographic structures [11-14]. For instance, a fishbone-like holographic structure from the interference by the direct and the backscattered electron wave packets has been identified experimentally [15]. This structure has been proposed as a particularly sensitive probe of the molecular structures [12]. Hence, how to decode the structural and dynamical information about the target from a given holographic structure has also attracted great attention. This has led to a novel approach for extracting the phase of the scattering amplitude of the signal wave, providing time-resolved imaging of ultrafast processes [16].

Nonetheless, the understanding of time-resolved photoelectron holography is still quite preliminary. Holographic patterns are usually understood within the strong-field approximation (SFA) [1,2], or semiclassical models in which the influence of the ionic Coulomb potential on the dynamics of the ionized electron is fully neglected [11,12]. Recently, however, the Coulomb potential has been found to play an important role in the photoelectron spectra, leading to, e.g., an unexpected low-energy structure [17-21] and even a zero-energy structure [22,23]. The Coulomb potential also modifies the holographic patterns, resulting in, e.g., the reduced fringe spacing in the spiderlike holographic structure [6,7] and the appearance of the clear backscattering holography due to the Coulomb focusing [11-13]. The physics behind the Coulomb effects is, however, poorly understood, which greatly hinders a comprehensive understanding of photoelectron holography and its potential applications in strong-field and attosecond physics.

Another structure caused by the interplay between the Coulomb potential and the laser field is a fan-shaped interference pattern that appears in two-dimensional PADs near the ionization threshold. This structure has been measured in several experiments $[6,24,25]$ and has been the topic of theoretical studies since the past decade [26-29]. Regardless, there is no direct explanation of how this pattern forms. Empirical rules for predicting the number of fringes have been given in [26,28], but this rule loses its efficacy as the 
laser intensity is increased [30]. Furthermore, in [26] the patterns were related to laser-dressed Kepler hyperbolae with neighboring angular momenta. However, the arguments in [26] are backed by classical-trajectory Monte Carlo (CTMC) computations, for which quantum interference is absent. This means that there is no direct evidence that the fanlike structure can be reproduced, or how it develops. Subsequently, the fan-shaped structure is reproduced with the Coulomb-Volkov approximation [27], for which the influence of the Coulomb potential is included in the final electron state, but not in the continuum propagation. Hence, it does not provide information on how the Coulomb potential changes the electron trajectories and only allows a vague explanation for how the patterns form by performing a comparison with the SFA. Therefore, a new theoretical method is required to reveal the underlying physics of the Coulomb effect on the fan-shaped interference patterns.

In this paper, we study the above-mentioned fan-shaped structure with a Coulomb quantum-orbit strong-field approximation (CQSFA) theory [31]. This newly developed approach exhibits a very good agreement with the result from the time-dependent Schrödinger equation (TDSE), and allows a direct assessment of quantum interference in terms of a few electron trajectories and their phase differences. We perform a detailed analysis of how the fan-shaped pattern forms, and, more importantly, show that it corresponds to a subcycle timeresolved holographic structure arising from the interference between the direct and the forward-scattered electron wave packets. This type of forward scattering is absent in the SFA, and corresponds to trajectories along which the electron is deflected by the Coulomb potential without undergoing a hard collision with the core. Due to the Coulomb potential, the phase associated with the forward-scattered trajectories is significantly changed. These distortions are angle dependent, and more dramatic for lower-energy photoelectrons, resulting in the specific fan-shaped structure. Thus, our work explains how the fan-shaped structure forms under the influence of Coulomb potential. Moreover, our work shows that the fan-shaped pattern can be used to extract information on the target structure, as, for a given set of parameters, the number of fringes differs for symmetric and antisymmetric bound states. Additionally, we analyze the electron ionization dynamics relevant to the fan-shaped structure and identify a clear signature of nonadiabatic tunneling.

This paper is organized as follows. In Sec. II, we briefly introduce the CQSFA theory and state the difference between our approach and other theoretical methods. Subsequently, in Sec. III, we present the CQSFA, SFA, and TDSE simulations of the two-dimensional PADs of atoms near the ionization threshold in a linearly polarized laser field and, furthermore, reveal the underlying physics of the Coulomb effect on the fan-shaped structure. Finally, in Sec. IV our conclusions are given. Atomic units (a.u.) are used throughout unless otherwise indicated.

\section{THEORETICAL METHODS}

The CQSFA theory [31] employed in this work describes ionization in terms of quantum orbits from the saddle-point evaluation of the ionization amplitude. Briefly, in the CQSFA theory, the initial state is a bound state $\left|\psi_{0}\left(t_{0}\right)\right\rangle=e^{i I_{p} t_{0}}\left|\psi_{0}\right\rangle$, and the final state is a continuum state $\left|\psi_{\mathbf{p}_{f}}(t)\right\rangle$ with momentum $\mathbf{p}_{f}$. This gives the ionization amplitude [2],

$$
M\left(\mathbf{p}_{f}\right)=-i \lim _{t \rightarrow \infty} \int_{-\infty}^{t} d t_{0}\left\langle\psi_{\mathbf{p}_{f}}(t)\left|\hat{U}\left(t, t_{0}\right) \hat{H}_{I}\left(t_{0}\right)\right| \psi_{0}\left(t_{0}\right)\right\rangle,
$$

where $\hat{U}\left(t, t_{0}\right)$ is the time-evolution operator of the Hamiltonian $\hat{H}(t)=\hat{\mathbf{p}}^{2} / 2+V(\hat{\mathbf{r}})+\hat{H}_{I}(t)$ with $\hat{H}_{I}(t)=-\hat{\mathbf{r}} \cdot \mathbf{E}(t)$ and the Coulomb potential $V(\hat{\mathbf{r}})$. Note that Eq. (1) is formally exact. Employing the Feynman path-integral formalism [32,33] and the saddle-point approximation [34,35], Eq. (1) becomes

$$
\begin{aligned}
M\left(\mathbf{p}_{f}\right) \propto & -i \lim _{t \rightarrow \infty} \sum_{s}\left\{\operatorname{det}\left[\frac{\partial \mathbf{p}_{s}(t)}{\partial \mathbf{r}_{s}\left(t_{0, s}\right)}\right]\right\}^{-1 / 2} e^{i S\left(\tilde{\mathbf{p}}_{s}, \mathbf{r}_{s}, t_{0, s}, t\right)} \\
& \times \mathcal{C}\left(t_{0, s}\right)\left\langle\mathbf{p}_{s}\left(t_{0, s}\right)+\mathbf{A}\left(t_{0, s}\right)\left|\hat{H}_{I}\left(t_{0, s}\right)\right| \psi_{0}\right\rangle
\end{aligned}
$$

where the term $\mathcal{C}\left(t_{0, s}\right)=\sqrt{2 \pi i /\left(\partial S\left(\tilde{\mathbf{p}}_{s}, \mathbf{r}_{s}, t_{0, s}, t\right) / \partial t_{0, s}\right)}$ is the prefactor, $\partial \mathbf{p}_{s}(t) / \partial \mathbf{r}\left(t_{0, s}\right)$ is related to the stability of the trajectory,

$$
S\left(\tilde{\mathbf{p}}, \mathbf{r}, t_{0}, t\right)=I_{p} t_{0}-\int_{t_{0}}^{t} d \tau\left[\dot{\mathbf{p}} \cdot \mathbf{r}(\tau)+\tilde{\mathbf{p}}^{2} / 2+V(\mathbf{r})\right]
$$

denotes the action, in which $I_{p}$ is the ionization potential, $\mathbf{p}$ is the canonical momentum, and $\tilde{\mathbf{p}}=\mathbf{p}+\mathbf{A}(\tau)$, with $t_{0}<\tau<t$, is the electron velocity. The index $s$ denotes the different orbits from three saddle-point equations:

$$
\begin{gathered}
{\left[\mathbf{p}_{0}+\mathbf{A}\left(t_{0}\right)\right]^{2} / 2+I_{p}=0,} \\
\dot{\mathbf{p}}(\tau)=-\nabla_{\mathbf{r}} V[\mathbf{r}(\tau)],
\end{gathered}
$$

and

$$
\dot{\mathbf{r}}(\tau)=\mathbf{p}(\tau)+\mathbf{A}(\tau),
$$

which are solved using an iteration scheme for any given final momentum [31] with the assumption that the electron is ionized by tunneling from $t_{0}$ to $t_{0}^{R}=\operatorname{Re}\left[t_{0}\right]$ and then moves to the detector with the real time [36,37]. In practice, we assume that the momentum of the electron is fixed during the tunneling ionization. In this case, the second term on the right-hand side of the action $S\left(\tilde{\mathbf{p}}, \mathbf{r}, t_{0}, t\right)$ is split into a part inside the barrier,

$$
\tilde{S}^{\text {in }}\left(\tilde{\mathbf{p}}, \mathbf{r}, t_{0}\right)=-\int_{t_{0}}^{t_{0}^{R}}\left[\tilde{\mathbf{p}}^{2} / 2+V(\mathbf{r})\right] d \tau
$$

with the tunneling trajectory $\mathbf{r}(\tau)=\int_{t_{0}}^{\tau}\left[\mathbf{p}\left(\tau^{\prime}\right)+\mathbf{A}\left(\tau^{\prime}\right)\right] d \tau^{\prime}[36]$ and a part outside the barrier,

$$
\tilde{S}^{\text {out }}\left(\tilde{\mathbf{p}}, \mathbf{r}, t_{0}, t\right)=-\int_{t_{0}^{R}}^{t} d \tau\left[\dot{\mathbf{p}} \cdot \mathbf{r}(\tau)+\tilde{\mathbf{p}}^{2} / 2+V(\mathbf{r})\right],
$$

with the ionization trajectory determined by Eq. (6).

Our simulations show that there are four types of trajectories for each photoelectron with any given final momentum [31]. This is consistent with Ref. [38], where this classification was first introduced. Here, we assume that the laser polarization is along the $z$ axis and the final momentum along the laser polarization $p_{f, z}>0$. For trajectories of type I, the tunneling exit $z_{0}>0$, and the electron moves directly towards the detector without returning to its parent ion. For the type II 

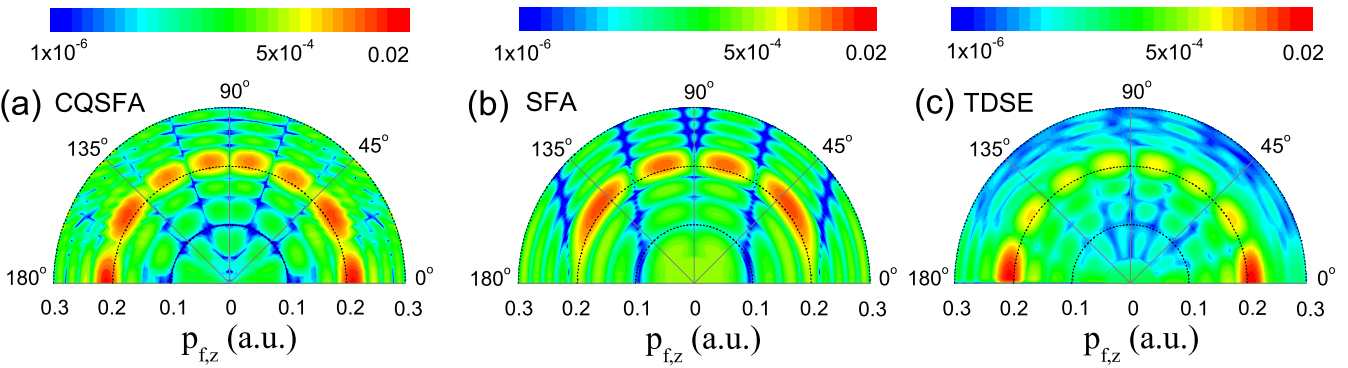

FIG. 1. Two-dimensional PADs of hydrogen atom $\left(I_{p}=0.5\right.$ a.u. $)$ near the ionization threshold in a linearly polarized laser field of intensity $I=2 \times 10^{14} \mathrm{~W} / \mathrm{cm}^{2}$ and wavelength $\lambda=800 \mathrm{~nm}$, for momenta $p_{f}<0.3$ a.u. Panels (a), (b), and (c) refer to CQSFA, SFA, and TDSE, respectively. The momentum component along the laser polarization direction is given by $p_{f, z}$. In (a) and (b), we have used $\mathbf{E}(t)=\hat{\mathbf{z}} E_{0} \sin \omega t$ over five cycles, while in (c) we have taken a long laser pulse $\mathbf{E}(t)=\hat{\mathbf{z}} E_{0} \sin \omega t \times f(t)$ with a trapezoidal profile $f(t)$ (up- and down-ramped over 2 cycles, constant over 8 cycles). The TDSE spectra have been computed employing a window operator of width $5 \times 10^{-4}$ a.u. as discussed in [46]. All panels have been normalized to the same range to facilitate a direct comparison.

and III trajectories, the tunneling exit $z_{0}<0$, meaning that the initial motion carries the electron away from the detector before it turns around and finally arrives with the stipulated momentum $\mathbf{p}_{f}$. A closer inspection shows that they are similar to Kepler hyperbolae to which a drift motion caused by the field is superimposed [26,38]. Trajectory types I and II are similar to the so-called "short" and "long" trajectories in the SFA theory. Trajectory type III is not found in the SFA and can be observed after the Coulomb potential is considered. In more strict terms, however, our previous results show that orbit II is twofold degenerate in the SFA, and that the presence of the Coulomb potential lifts this degeneracy [31,39].

Finally, there exists an additional trajectory type, denoted as IV. For this type of orbit, although the tunnel exit points towards the detector, the electron is driven back to the core by the laser field, then goes around the core, and finally moves towards the detector. With increasing photoelectron final momentum, the shortest distance between the electron and the core decreases [31]. This distance can be smaller than the tunnel exit. In this case, this type of trajectory corresponds to a rescattering event. It is noteworthy that our numerical simulations show that only type I-III trajectories are needed for obtaining converged photoelectron spectra in the low-energy region [31]. Illustrations of these types of trajectories are provided in our previous publication [31].

Conceptually, the CQSFA differs from the Coulombcorrected SFA (CCSFA) theory [38,40], the Eikonal-Volkov approximation (EVA) [41] and the analytical $R$-matrix (ARM) method [42,43], which are the most widespread Coulombcorrected strong-field approaches. While the EVA is derived from a laser-dressed Wentzel-Kramers-Brillouin (WKB) approach in the limit of small scattering angles, and the CCSFA constructs its trajectories recursively starting from the Coulomb-free trajectories used in the SFA, the CQSFA is derived using path-integral methods, which are applied to the full time-evolution operator. This leads to different photoelectron transition amplitudes, e.g., the $\dot{\mathbf{p}} \cdot \mathbf{r}(\tau)$ term in the action of Eq. (3) which is absent in the CCSFA $[38,40]$. This term is important for obtaining correct interference patterns, as found in our previous publication [31] for ATI spectra and in Ref. [44] for ATI PADs. The ARM treats hard collisions differently, by imposing a spatial boundary. Inside the boundary, the whole core dynamics are incorporated and the field is treated perturbatively, while outside the barrier the EVA is employed.

From the implementation viewpoint, there are also differences as the CCSFA solves the direct problem of seeking the final momentum for a given initial momentum, while the CQSFA focuses on the inverse problem. An important consequence is that, while for both the CQSFA and the CCSFA there are four main types of interfering orbits, the number of contributed trajectories is different for the two approaches. In fact, sampling in the CCSFA is implemented to obtain a large number of orbits and then these trajectories are binned according to their final momenta. Thus, in practice, there is a huge amount of electron trajectories in the CCSFA, while, in the CQSFA, one contributed trajectory of each relevant type suffices. For example, for each photoelectron in the low-energy region, three trajectories within a driving-field cycle are needed for obtaining converged PADs. Therefore, by analyzing the phase difference between these few orbits, we can directly understand how the interference patterns are formed and how the Coulomb potential influences this interference.

In this work, we study the ionization of the hydrogen atom in a linearly polarized laser field of intensity $I=2 \times$ $10^{14} \mathrm{~W} / \mathrm{cm}^{2}$ and wavelength $\lambda=800 \mathrm{~nm}$. Unless otherwise stated, the initial state is taken as the ground state of the hydrogen atom, $\psi_{0}(\mathbf{r})=\left\langle\mathbf{r} \mid \psi_{0}\right\rangle=e^{-r} / \sqrt{\pi}$. In this case, the form factor in Eq. (2) becomes related to the atomic dipole moment and can be simplified as $\left\langle\mathbf{p}\left(t_{0}\right)+\mathbf{A}\left(t_{0}\right)\right|-\mathbf{r}$. $\mathbf{E}\left(t_{0}\right)\left|\psi_{0}\right\rangle \sim E\left(t_{0}\right) \tilde{p}_{0 z}$ in a linearly polarized laser field along the $z$ axis [45], where $\tilde{p}_{0 z}$ is the component of the momentum $\tilde{\mathbf{p}}_{0}=\mathbf{p}\left(t_{0}\right)+\mathbf{A}\left(t_{0}\right)$ along the laser polarization direction.

\section{RESULTS AND DISCUSSION}

Figures 1(a) and 1(b) exhibit the two-dimensional PADs near the ionization threshold computed for the hydrogen atom in a linearly polarized laser field with the CQSFA and the SFA, respectively. As a benchmark, we take the ab initio TDSE calculation shown in Fig. 1(c), which is solved using the freely available software QPROP [46]. Our results show significant changes in the PAD for the CQSFA, in comparison with the SFA simulation. Indeed, there are eight clear peaks in the first 

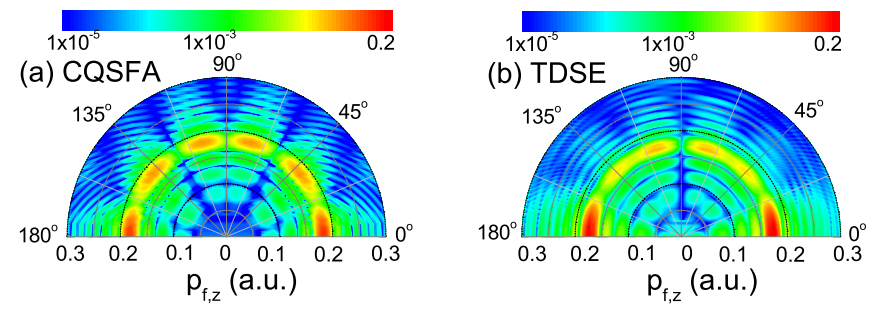

FIG. 2. Same as Figs. 1(a) and 1(c), but with the different laser intensity of $I=5 \times 10^{13} \mathrm{~W} / \mathrm{cm}^{2}$.

ATI ring at the momentum $p_{f} \sim 0.2$ a.u., while only four peaks are found in the corresponding SFA simulations. Moreover, in Fig. 1(a) a clear radial fanlike pattern is present between the threshold region and the onset of the first ATI ring, which completely disappears in Fig. 1(b). The overall interference pattern in CQSFA exhibits a very good agreement with the TDSE simulations in Fig. 1(c), reflecting the significant role of the Coulomb potential on strong-field ionization, which is consistent with previous publications $[26,27]$. Note that there are plenty of examples in the literature, both theoretical and experimental, for which this structure is reproduced in different regimes, such as the multiphoton ionization regime or the deeply tunneling ionization regime (e.g., low-frequency, mid-IR regime). In these regimes, the number of peaks of the fan-shaped structure is changed, but not the pattern $[26,28]$. Indeed, we have also simulated the fan-shaped structure with the CQSFA theory using different laser parameters, for example, $I=5 \times 10^{13} \mathrm{~W} / \mathrm{cm}^{2}$ (Keldysh parameter $\gamma=1.5$ [1]; see Fig. 2). In this figure, there are only six peaks left in the first ATI ring at the momentum $p_{f} \sim 1.8$ a.u, but the fan-shaped structure is still present. Moreover, the overall interference pattern in CQSFA is qualitatively consistent with the TDSE simulations in Fig. 2(b), including the correct number of the peaks. This latter feature has turned out to be a challenge in Coulomb corrected methods. For instance, the CCSFA method fails to reproduce the correct number of the maxima in the TDSE result (see discussion in Ref. [40]). Only very recently, this has been independently achieved in [44].

It is worth noting that there is a slight quantitative discrepancy between CQSFA and TDSE in the ionization amplitudes. For example, Figs. 1 and 2 show that the TDSE yield of the first ATI ring decays much faster with increasing scattering angle
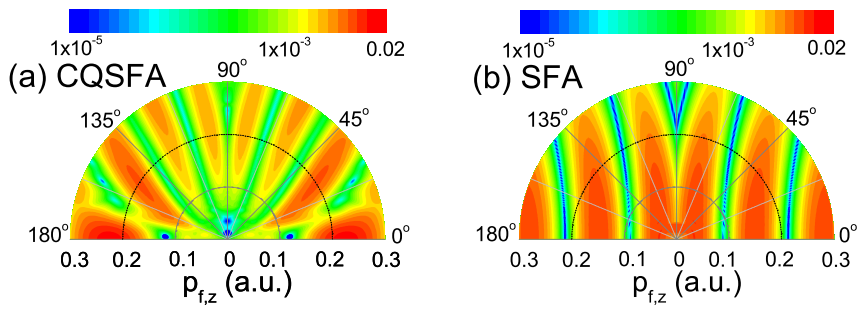

FIG. 4. Same as Figs. 1(a) and 1(b), but calculated with quantum orbits occurred in one optical cycle.

than for the CQSFA (see also Fig. 3 for a closer look at the first ATI ring). Possible reasons are that the initial electronic wave packet in the TDSE has an initial momentum and position spread, while all CQSFA orbits are located at the tunnel exit. The initial spread of the electronic wave packet has been shown to be necessary for a good quantitative agreement with the TDSE in the context of high-order harmonic generation (HHG) [53-56]. This initial spread seems to be very important for strong-field ionization as well, as recent phase-space studies revealed that orbit-based methods that incorporate this spread exhibit a nearly quantitative agreement with the TDSE, even if tunnel ionization is not properly accounted for [57]. In addition, in the very low-energy region of the spectra, the CQSFA simulation exhibits a marked discrepancy with respect to the TDSE result. The main reason is that, because orbits II and III may turn around the core before being detected, a photoelectron with very low energy is greatly sensitive to the Coulomb potential. In the presence of both the field and the potential, this renders orbits II and III highly irregular and hence difficult to compute. Nevertheless, in this work we focus on how the Coulomb potential leads to the generation of the fanlike structure by analyzing quantum orbits from the CQSFA and the interference pattern in CQSFA qualitatively exhibits a very good agreement with the TDSE simulations.

In Fig. 4 we consider only the interference of the orbits within one driving-field cycle, i.e., the intracycle interference [29]. The CQSFA outcome [Fig. 4(a)] exhibits eight interference stripes, which, near the ionization threshold, roughly point to zero momentum, showing a divergent structure. In contrast, for the SFA simulations in Fig. 4(b), there are only six approximately vertical interference stripes, which bend as the transverse momentum increases. If quantum-
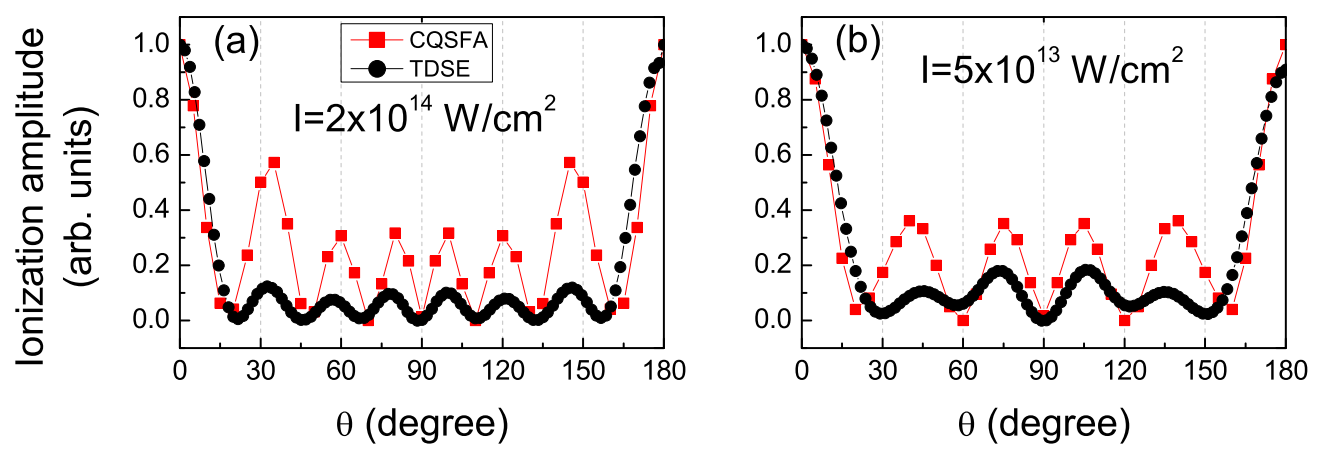

FIG. 3. (a) and (b) Angular photoelectron distributions of the first ATI ring shown in Figs. 1 and 2, respectively. The square curve in red and the circle curve in black refer to the CQSFA and TDSE solutions, respectively. The amplitude has been normalized to the maximum value. 


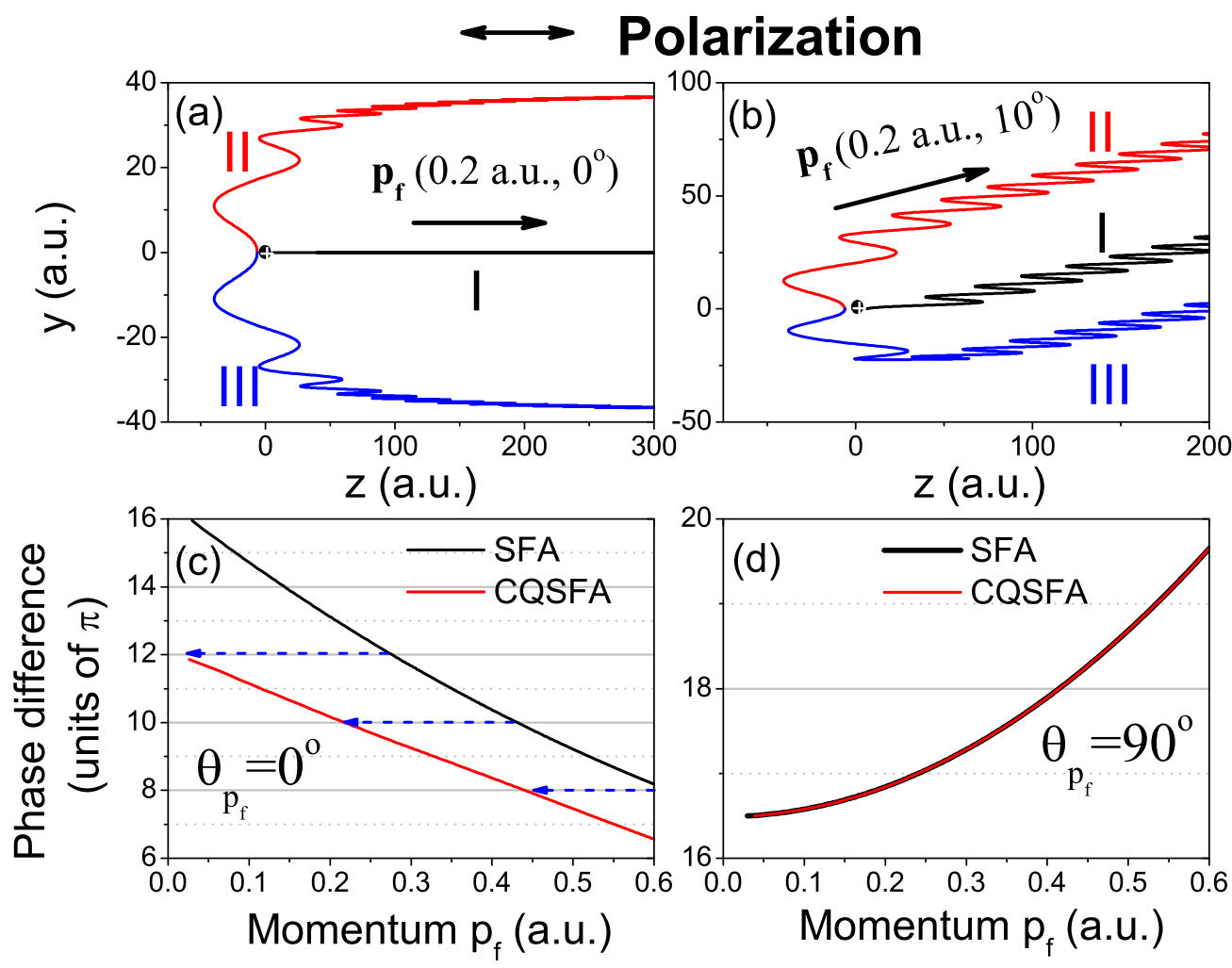

FIG. 5. (a) and (b) Illustration of three orbits from the CQSFA theory in the $y z$ plane for electrons with fixed final momentum $p_{f}=0.2$ a.u. along the $0^{\circ}$ and $10^{\circ}$ directions with respect to the laser polarization, respectively. The laser polarization is along the $z$ axis. The black circle at the position $(0,0)$ denotes the nucleus. (c) and (d) Phase difference between orbits I and II as a function of $p_{f}$ along $0^{\circ}$ and $90^{\circ}$ directions with respect to the laser polarization, respectively. The blue dashed arrows denote the shift of the positions of the interference maxima.

orbit contributions from other optical cycles are also added coherently, intercycle interference [29] forms characteristic ATI rings centered around zero momentum. The modulation between the intracycle and intercycle interference results in the clear eight peaks in the first ATI ring in CQSFA and the four ATI peaks in SFA, just as illustrated in Figs. 1(a) and 1(b), respectively. Moreover, the divergent structure in Fig. 4(a) corresponds to the fanlike pattern shown in the PADs in Fig. 1(a). Thus, the difference between Figs. 1(a) and 1(b) stems from the influence of the Coulomb potential on the intracycle interference.

More insight can be gained by analyzing the positions of the interference stripes in the PADs. In the CQSFA theory, the amplitude of the intracycle interference in the low-energy region is mainly determined by three quantum orbits [31]. Figures 5(a) and 5(b) depict these orbits in the $y z$ plane for electrons with fixed final momentum $p_{f}=0.2 \mathrm{a}$.u. along the $0^{\circ}$ and $10^{\circ}$ directions with respect to the laser polarization, respectively. For orbit I, the electron moves directly towards the detector without returning to the parent ion. In contrast, for orbits II and III, the electron will turn around the core and then move to the detector along Kepler hyperbolae to which a quiver motion caused by the laser field is superimposed $[26,38]$. Therefore, the patterns in Fig. 4(a) correspond to a holographic structure from the interference between the direct trajectories and forward-scattered trajectories, which are deflected by the core but do not undergo hard collisions. Orbits I and II are similar to the short and long trajectories in SFA [11], while orbit III is not found in the SFA and is observed after the Coulomb potential is considered $[6,38]$. If the final momentum is along the laser polarization [Fig. 5(a)], orbits II and III are symmetric with respect to the polarization direction and have the same amplitudes. With increasing scattering angle $\theta_{\mathbf{p}_{f}}$, orbit III will experience a stronger attraction from the Coulomb potential, leading to a larger deflection [see, e.g., Fig. 5(b)]. Due to Coulomb defocusing, the amplitude of orbit III decreases significantly with increasing scattering angle especially for low-energy photoelectrons [47], while the contributions from orbits I and II become dominant. This results in well-defined reference and probe signals in the holographic patterns: orbits I and II, respectively.

Hence, we will focus on the phase difference between orbits I and II, $\Delta \Phi=\Phi_{\mathrm{I}}-\Phi_{\mathrm{II}}$, which is directly related to the interference pattern and is displayed in Figs. 5(c) and 5(d) as a function of the final electron momentum, for parallel and perpendicular scattering angles, respectively. A similar analysis has been employed in our previous publications [31,48]. For $\theta_{\mathbf{p}_{f}}=0^{\circ}$, the phase difference decreases if the Coulomb potential is incorporated. This shifts the interference maxima in the CQSFA spectra towards lower energies, in comparison with their SFA counterparts (see the blue dashed arrows). Physically, this happens because, in comparison with orbit I, orbit II accumulates a larger positive phase contribution from the Coulomb potential as it passes by the core, $-\int_{t^{\prime}}^{t} V[\mathbf{r}(\tau)] d \tau$ [see Eq. (2)]. With increasing $\theta_{\mathbf{p}_{f}}$, the above-mentioned shift becomes smaller and is almost negligible for perpendicular emission [Fig. 5(d)]. This is not surprising since for $\theta_{\mathbf{p}_{f}}=90^{\circ}$ orbits I and II are symmetric with respect to the $y$ axis. Thus, 
(a)

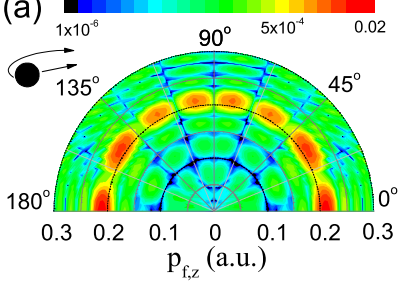

(b)

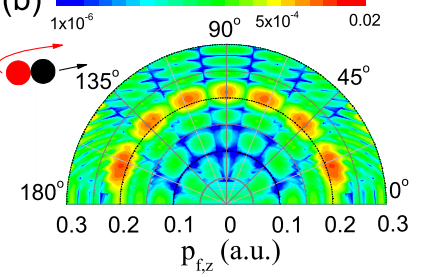

FIG. 6. (a) Same as Fig. 1(a) for the initial state of hydrogen atom $\psi_{0}(\mathbf{r})=e^{-r} / \sqrt{\pi}$, and (b) for the initial state of $\psi_{0}^{(a)}(\mathbf{r}) \sim e^{-r} \cos \theta$. The schematic diagram on the left side denotes the orbital symmetry of the initial state and the interference trajectories. For more details, see the text.

the influence of the Coulomb potential on the two orbits is the same. A similar result has been reported in the study of the interference carpets in ATI [49]. Therefore, more interference stripes will appear in the low-energy region for the CQSFA, in agreement with Fig. 4(a). Furthermore, the shift in $\Delta \Phi$ is more significant for smaller momenta [Fig. 5(c)] as, in this case, the electron will need a longer time to leave the core region. This will result in a larger positive phase contribution from the Coulomb potential and a larger decrease in $\Delta \Phi$. Therefore, the interference maxima in the photoelectron spectra will shift more dramatically for smaller momenta. A similar behavior is observed for other emission angles $\theta_{\mathbf{p}_{f}}$, leading to the fanlike structures in Figs. 1(a) and 4(a).

In the discussion above, we have assumed that the electron is freed from the ground state of hydrogen atom with spherical symmetry, $\psi_{0}(\mathbf{r})=e^{-r} / \sqrt{\pi}$. Indeed, we find that the symmetry of the initial electronic state also significantly influences the

fan-shaped structure. If, instead, we employ an antisymmetric initial state, e.g., $\psi_{0}^{(a)}(\mathbf{r})=\left\langle\mathbf{r} \mid \psi_{0}^{(a)}\right\rangle \sim e^{-r} \cos \theta$, and keep the same binding energy ( 0.5 a.u. $)$ for comparison, the tunnel matrix element in Eq. (2) becomes $\left\langle\mathbf{p}\left(t_{0}\right)+\mathbf{A}\left(t_{0}\right)\right|-\mathbf{r}$. $\mathbf{E}\left(t_{0}\right)\left|\psi_{0}^{(a)}\right\rangle \sim E(t) \tilde{p}_{0 z}^{2}$. Figure 6 shows the CQSFA simulations of the two-dimensional PADs near the ionization threshold in a linearly polarized laser field for the two kinds of initial states. As one can see, for the initial state with spherical symmetry, there are eight peaks in the first ATI ring, while for the state with antisymmetry, only seven peaks can be observed in the first ATI ring. The reason for the divergence is that for the state with antisymmetry, there is an additional phase difference of $\pi$ between the direct trajectory and the forwardscattered trajectory during the tunneling ionization [50] (see the schematic diagram in Fig. 6). This results in the opposite interference patterns for the two initial states with different symmetries: The peaks shown in Fig. 6(a) are suppressed in the PAD in Fig. 6(b), while the ionization suppression in Fig. 6(a) becomes enhanced in Fig. 6(b). Therefore, our simulation shows that the fan-shaped pattern can be used to extract information about the target's structure by analyzing two-dimensional PADs. Note that our results are similar to the finding in [28], in which an empirical dominant orbital angular momentum related to the fan-shaped pattern changes for initial $s$ and $p$ states. In contrast, in a CTMC simulation [26], it is found that the dominant angular momentum in the fanlike pattern is virtually independent of the atomic species.

Finally, Figs. 7(a) and 7(b) show the tunneling time of the photoelectrons from CQSFA and SFA for orbits I and II, respectively, for a specific angle $\theta_{\mathbf{p}_{f}}=0^{\circ}$. Due to the ionization by tunneling, the time $t_{0}$ becomes complex [2], and $\operatorname{Im}\left[t_{0}\right]>0$
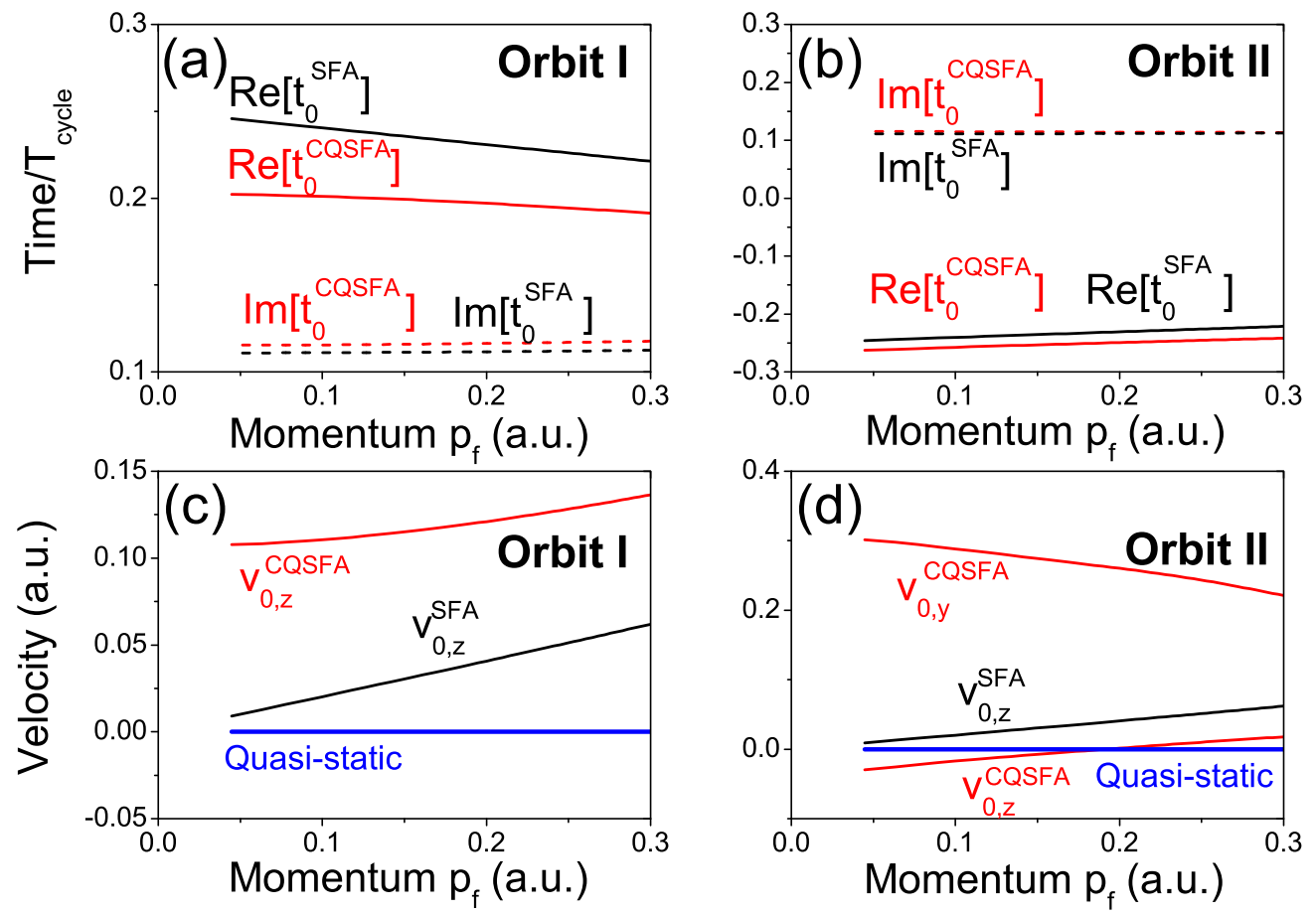

FIG. 7. (a) and (b) The real part (solid lines) and imaginary part (dashed lines) of the tunneling time for electrons with $\theta_{\mathbf{p}_{f}}=0^{\circ}$ in CQSFA (red) and in SFA (black). (c) and (d) The corresponding initial velocity at the tunneling exit. The quasistatic expected velocity is denoted by the blue lines. (a) and(c), orbit I; (b) and (d), orbit II. 
can be related to the tunneling time through the potential barrier. For each kind of orbit, the photoelectrons are initially ionized within a temporal window of about $0.02 T_{\text {cycle }}(\sim 60$ attoseconds), and the orbits I and II originate from the adjacent quarter cycles of the laser pulse. Therefore, the subcycle fan-shaped structure has recorded attosecond time-resolved electronic dynamics. In comparison with the SFA, for a given final momentum $\mathbf{p}_{f}, \operatorname{Re}\left(t_{0}\right)$ in the CQSFA approaches the driving-field crossing $(t=0)$ or its crest $\left(t=-0.25 T_{\text {cycle }}\right)$ for orbit I or II, respectively. This increases (decreases) the initial field-dressed momentum $\mathbf{p}_{0}$ for orbit I (orbit II). Along orbit I, the electron experiences a deceleration in the Coulomb potential as it moves towards the detector, while, for orbit II, the electron is accelerated significantly along the polarization direction due to the interplay of the Coulomb potential and the laser field [31,36]. In Figs. 7(c) and 7(d) we illustrate the change of the initial velocity $\mathbf{v}_{0}=\mathbf{p}_{0}+\mathbf{A}\left(\operatorname{Re}\left[t_{0}\right]\right)$ at the tunneling exit. Both CQSFA and SFA simulations significantly deviate from the adiabatic tunneling theory (blue lines in Fig. 7), in which the electron is assumed to begin its journey in the continuum with vanishing velocity $[1,51,52]$.

\section{CONCLUSIONS}

In summary, we have performed a detailed analysis of the low-energy fanlike structure observed in PADs using a CQSFA theory, in which only a few electron trajectories are required to describe strong-field ionization, and which poses no restriction upon the scattering angle. We show that this structure constitutes a subcycle time-resolved holographic pattern from the interference of direct electron trajectories and forwardscattered trajectories that are deflected, but do not undergo hard collisions with the core. We go beyond existing studies by providing direct and in-depth evidence of how the Coulomb potential alters the phase of the forward-scattered trajectories. This affects different scattering angles and electron momenta unequally, leading to the above-mentioned fanlike structure. Moreover, our work shows that the fan-shaped pattern can be used to extract information on the target structure, based on the fact that the number of fringes in the PADs depends strongly on the symmetry of the electronic bound state.

This physical interpretation of the fan-shaped structure differs from those presented in the literature. In [26], the emphasis is placed on the fact that the fan-shaped structure corresponds to a single specific Legendre polynomial. The structures are then related to Ramsauer-Townsend fringes using indirect arguments. Furthermore, the laser-dressed Kepler hyperbolae with neighboring angular momenta that are associated with the intracycle fringes would correspond to type II trajectories in the present notation. However, due to the method being classical and thus not accounting for quantum interference, no direct evidence that the fanlike structure can be reproduced is provided. A similar line of argument is used in [28] for a very different parameter range, namely the multiphoton regime. Therein, it is stated that the fan-shaped structure is caused by the quantum state with an empirical dominant orbital momentum quantum number $l$, which is the electron's main pathway into the continuum.

One should also note that the fan-shaped structure and the spiderlike structure reported in Refs. [6,7] are very distinct holographic patterns, formed by the interference of different types of contributing trajectories in very distinct energy regions. While the fan-shaped structure stems from the interference of orbits I and II, the spiderlike structure stems from the interference of orbits II and III. This discrepancy is related to the fact that the wave packet of orbit III is strongly deflected by the core. With the increase of scattering angle, a significant decrease in the probability density of orbit III rules out its contributing to the fan-shaped structure. On the other hand, the probability density of orbit III is concentrated along the polarization axis, which is also the momentum region occupied by the spiderlike structure. There is also an "inner" spiderlike holographic structure, which occurs near the ionization threshold. This structure was reported in Ref. [14], and has been attributed to the interference between type II and type III orbits undergoing multiple recollisions. Since the ionization events associated with the latter orbits are less frequent than the standard type I and II orbits, they are not prevalent in the fan-shaped structure studied in this work. For details on the high-energy spiderlike structure see our recent article [39].

In addition, studies employing the closely related CCSFA method focus either on interference patterns at much higher photoelectron energies [40], or on the low-energy structure (LES) [38], whose nature is found to be classical and related to Coulomb focusing. The fan-shaped structure is attributed to the interference of all four types of trajectories, while in our present work we find that it involves mainly trajectories I and II. Typically, intracycle interference near the threshold is viewed as a double slit type of interference between trajectories reaching the detector directly and those starting in the opposite side. This is a well-known effect within the SFA [29], for which deflection by the Coulomb potential is neglected. Since, however, the degeneracy of orbit II is lifted by the Coulomb potential [31], it was not at all clear that the main contribution to the fan-shaped structure would still be caused by only two types of trajectories.

It is also noteworthy that, overall, the CQSFA exhibits a very good agreement with the TDSE. This includes the number of fringes and substructure in the fan-shaped structure. The TDSE yield, however, decays faster with regard to the deflection angle. This may be due to the initial momentum and position spread that exists for the TDSE, but is absent for the CQSFA. In fact, recent studies on HHG [53-56] and strong-field ionization [57] indicate that this spread is necessary for a quantitative agreement with the TDSE.

Finally, a great advantage of the CQSFA is that it provides a transparent picture in terms of electron orbits leaving its parent ion and reaching the detector, whose contributions can be switched on and off at will and directly influence the resulting patterns. This means that the present method can be applied to the understanding of Coulomb effects on other holographic patterns, e.g., the well-known reduced fringe spacing in the spiderlike holographic structure [6,7].

\section{ACKNOWLEDGMENTS}

We thank Professor Wilhelm Becker and Professor Xue-Bin Bian for many useful discussions. This work is supported by 
the National Basic Research Program of China (Grant No. 2013CB922201), the NNSF of China (Grants No. 11374329,
No. 11334009, No. 11474321, and No. 11527807), and the UK EPSRC (Grant No. EP/J019240/1).
[1] L. V. Keldysh, Zh. Eksp. Teor. Fiz. 47, 1945 (1964) [Sov. Phys. JETP 20, 1307 (1965)]; F. H. M. Faisal, J. Phys. B 6, L89 (1973); H. R. Reiss, Phys. Rev. A 22, 1786 (1980).

[2] W. Becker, F. Grasbon, R. Kopold, D. B. Milošević, G. G. Paulus, and H. Walther, Adv. At. Mol. Opt. Phys. 48, 35 (2002).

[3] F. Lindner, M. G. Schätzel, H. Walther, A. Baltuška, E. Goulielmakis, F. Krausz, D. B. Milosevic, D. Bauer, W. Becker, and G. G. Paulus, Phys. Rev. Lett. 95, 040401 (2005).

[4] M. Meckel, A. Staudte, S. Patchkovskii, D. M. Villeneuve, P. B. Corkum, R. Dörner, and M. Spanner, Nat. Phys. 10, 594 (2014).

[5] X. H. Xie, Phys. Rev. Lett. 114, 173003 (2015).

[6] Y. Huismans et al., Science 331, 61 (2010).

[7] Y. Huismans, A. Gijsbertsen, A. S. Smolkowska, J. H. Jungmann, A. Rouzée, P. S. W. M. Logman, F. Lépine, C. Cauchy, S. Zamith, T. Marchenko, J. M. Bakker, G. Berden, B. Redlich, A. F. G. van der Meer, M. Yu. Ivanov, T.-M. Yan, D. Bauer, O. Smirnova, and M. J. J. Vrakking, Phys. Rev. Lett. 109, 013002 (2012).

[8] M. Meckel et al., Science 320, 1478 (2008).

[9] M. Li, X. Sun, X. Xie, Y. Shao, Y. Deng, C. Wu, Q. Gong, and Y. Liu, Sci. Rep. 5, 8519 (2015).

[10] D. Gabor, Nature (London) 161, 777 (1948).

[11] X. B. Bian, Y. Huismans, O. Smirnova, K.-J. Yuan, M. J. J. Vrakking, and A. D. Bandrauk, Phys. Rev. A 84, 043420 (2011).

[12] X.-B. Bian and A. D. Bandrauk, Phys. Rev. Lett. 108, 263003 (2012).

[13] X.-B. Bian and A. D. Bandrauk, Phys. Rev. A 89, 033423 (2014).

[14] D. D. Hickstein, P. Ranitovic, S. Witte, X.-M. Tong, Y. Huismans, P. Arpin, X. Zhou, K. E. Keister, C. W. Hogle, B. Zhang, C. Ding, P. Johnsson, N. Toshima, M. J. J. Vrakking, M. M. Murnane, and H. C. Kapteyn, Phys. Rev. Lett. 109, 073004 (2012).

[15] M. Haertelt, X.-B. Bian, M. Spanner, A. Staudte, and P. B. Corkum, Phys. Rev. Lett. 116, 133001 (2016).

[16] Y. M. Zhou, O. I. Tolstikhin, and T. Morishita, Phys. Rev. Lett. 116, 173001 (2016).

[17] C. I. Blaga, F. Catoire, P. Colosimo, G. G. Paulus, H. G. Muller, P. Agostini, and L. F. Dimauro, Nat. Phys. 5, 335 (2009).

[18] W. Quan, Z. Lin, M. Wu, H. Kang, H. Liu, X. Liu, J. Chen, J. Liu, X. T. He, S. G. Chen, H. Xiong, L. Guo, H. Xu, Y. Fu, Y. Cheng, and Z. Z. Xu, Phys. Rev. Lett. 103, 093001 (2009).

[19] W. Becker et al., J. Phys. B 47, 204022 (2014).

[20] C. Y. Wu, Y. D. Yang, Y. Q. Liu, Q. H. Gong, M. Y. Wu, X. Liu, X. L. Hao, W. D. Li, X. T. He, and J. Chen, Phys. Rev. Lett. 109, 043001 (2012).

[21] W. Becker and D. B. Milošević, J. Phys. B 48, 151001 (2015).

[22] J. Dura et al., Sci. Rep. 3, 2675 (2013).

[23] W. Quan et al., Sci. Rep. 6, 27108 (2016).

[24] A. Rudenko et al., J. Phys. B 37, L407 (2004).

[25] C. M. Maharjan et al., J. Phys. B 39, 1955 (2006).

[26] D. G. Arbó, S. Yoshida, E. Persson, K. I. Dimitriou, and J. Burgdörfer, Phys. Rev. Lett. 96, 143003 (2006); D. G. Arbó, K. I. Dimitriou, E. Persson, and J. Burgdörfer, Phys. Rev. A 78, 013406 (2008).
[27] D. G. Arbó, J. E. Miraglia, M. S. Gravielle, K. Schiessl, E. Persson, and J. Burgdörfer, Phys. Rev. A 77, 013401 (2008).

[28] Z. Chen, T. Morishita, A. T. Le, M. Wickenhauser, X. M. Tong, and C. D. Lin, Phys. Rev. A 74, 053405 (2006).

[29] D. G. Arbó, K. L. Ishikawa, K. Schiessl, E. Persson, and J. Burgdörfer, Phys. Rev. A 81, 021403(R) (2010); D. G. Arbó et al., Nucl. Instrum. Methods B 279, 24 (2012).

[30] T. Marchenko, H. G. Muller, K. J. Schafer, and M. J. J. Vrakking, J. Phys. B 43, 095601 (2010).

[31] X. Y. Lai, C. Poli, H. Schomerus, and C. Figueira de Morisson Faria, Phys. Rev. A 92, 043407 (2015).

[32] H. Kleinert, Path Integrals in Quantum Mechanics, Statistics, Polymer Physics, and Financial Markets (World Scientific, Singapore, 2009).

[33] D. B. Milošević, J. Math. Phys. 54, 042101 (2013).

[34] C. Figueira de Morisson Faria, H. Schomerus, and W. Becker, Phys. Rev. A 66, 043413 (2002).

[35] R. Kopold, W. Becker, and M. Kleber, Opt. Commun. 179, 39 (2000).

[36] S. V. Popruzhenko and D. Bauer, J. Mod. Opt. 55, 2573 (2008).

[37] S. V. Popruzhenko, J. Phys. B 47, 204001 (2014).

[38] T.-M. Yan, S. V. Popruzhenko, M. J. J. Vrakking, and D. Bauer, Phys. Rev. Lett. 105, 253002 (2010).

[39] A. S. Maxwell, A. Al-Jawahiry, T. Das, and C. Figueira de Morisson Faria, Phys. Rev. A (to be published), arXiv:1705.1518.

[40] T.-M. Yan and D. Bauer, Phys. Rev. A 86, 053403 (2012).

[41] O. Smirnova, M. Spanner, and M. Ivanov, Phys. Rev. A 77, 033407 (2008).

[42] L. Torlina and O. Smirnova, Phys. Rev. A 86, 043408 (2012).

[43] J. Kaushal and O. Smirnova, Phys. Rev. A 88, 013421 (2013).

[44] N. I. Shvetsov-Shilovski, M. Lein, L. B. Madsen, E. Räsänen, C. Lemell, J. Burgdörfer, D. G. Arbó, and K. Tőkési, Phys. Rev. A 94, 013415 (2016).

[45] D. B. Milošević, G. G. Paulus, D. Bauer, and W. Becker, J. Phys. B 39, R203 (2006).

[46] D. Bauer and P. Koval, Comput. Phys. Commun. 174, 396 (2006); see also [http://www.qprop.de].

[47] Note that in comparison with the low-energy photoelectron in the fanlike structure, the decrease of the amplitude of orbit III with the scattering angle is relatively slow for the high-energy photoelectron, e.g., in the well-known spiderlike structure [6,7], because the influence of the Coulomb potential on the high-energy electron is relatively weak. Our recent work with the CQSFA method [39] clearly shows that the spiderlike structure is from the interference between oribts II and III, in good agreement with the previous publications [6,7].

[48] X. Y. Lai and C. Figueira de Morisson Faria, Phys. Rev. A 88, 013406 (2013).

[49] Ph. A. Korneev, S. V. Popruzhenko, S. P. Goreslavski, T.-M. Yan, D. Bauer, W. Becker, M. Kübel, M. F. Kling, C. Rödel, M. Wünsche, and G. G. Paulus, Phys. Rev. Lett. 108, 223601 (2012).

[50] D. B. Milošević, E. Hasović, M. Busuladžić, A. GazibegovićBusuladžić, and W. Becker, Phys. Rev. A 76, 053410 (2007).

[51] O. Pedatzur et al., Nat. Phys. 11, 815 (2015). 
[52] M. Y. Ivanov, M. Spanner, and O. Smirnova, J. Mod. Opt. 52, 165 (2005).

[53] M. B. Gaarde and K. J. Schafer, Phys. Rev. A 65, 031406(R) (2002).

[54] J. A. Pérez-Hernández, J. Ramos, L. Roso, and L. Plaja, Laser Phys. 20, 1044 (2010).
[55] J. Wu, B. B. Augstein, and C. Figueira de Morisson Faria, Phys. Rev. A 88, 023415 (2013).

[56] J. Wu, B. B. Augstein, and C. Figueira de Morisson Faria, Phys. Rev. A 88, 063416 (2013).

[57] C. Zagoya, J. Wu, M. Ronto, D. V. Shalashilin, and C. Figueira de Morisson Faria, New J. Phys. 16, 103040 (2014). 